\title{
Fast Design Optimization of UWB Antenna with WLAN Band-Notch
}

\author{
Slawomir Koziel, Adrian Bekasiewicz \\ Engineering Optimization \& Modeling Center \\ Reykjavik University \\ Reykjavik, Iceland \\ koziel@ru.is, bekasiewicz@ru.is
}

\author{
Selvakumar Ulaganathan, Tom Dhaene \\ Department of Information Technology \\ Ghent University, Gent, Belgium \\ selvakumar.ulaganathan@intec.ugent.be, \\ tom.dhaene@intec.ugent.be
}

\begin{abstract}
In this paper, a methodology for rapid design optimization of an ultra-wideband (UWB) monopole antenna with a lower WLAN band-notch is presented. The band-notch is realized using an open loop resonator implemented in the radiation patch of the antenna. Design optimization is a two stage process, with the first stage focused on the design of the antenna itself, and the second stage aiming at identification of the appropriate dimensions of the resonator with the purpose of allocating the band-notch in the desired frequency range. Both optimization stages are realized using surrogate-based optimization involving variable-fidelity electromagnetic (EM) simulation models as well as an additive response correction (first stage), and sequential approximate optimization (second stage). The final antenna design is obtained at the CPU cost corresponding to only 23 high-fidelity EM antenna simulations.
\end{abstract}

Keywords - UWB antennas; band-notch; simulation-driven design; EM-driven design; surrogate-based optimization; variable-fidelity simulations

\section{INTRODUCTION}

Ultra-wideband (UWB) technology has become extremely popular due to its many advantages, including high-speed data rate, low power consumption, as well as low cost [1]. UWB systems are typically utilized for short-range indoor communications. They are particularly attractive for applications such as wireless personal area networks [2], wireless peripherals [3], real time location systems [4], or medical imaging [5]. Antenna operating within a frequency range from $3.1 \mathrm{GHz}$ to $10.6 \mathrm{GHz}$ is a fundamental component of UWB system. The requirements upon modern antenna structures for UWB communication include, among others, small size [6], increased immunity to signal fading [6], or multiband operation [1].

An important issue with UWB systems is possible electromagnetic interference with other standards such as WLAN (5.15 to $5.24 \mathrm{GHz}$, and $5.75-5.85 \mathrm{GHz}$ ) or WiMAX (3.3-3.8 GHz). Avoiding these interferences requires rejecting certain sub-bands of the UWB range [7]. Thus, design of antenna structures that exhibit filtering properties at selected frequencies is of high interest. A considerable research effort has been focused on methods for realizing band-notch functionality. Some of the available techniques include slots cut in the radiation patch [8], the use of open-loop resonators [9], split-ring resonators [10], or the open-ended slots [11]. Regardless of the particular technique of implementing bandnotches, an important design challenge is adjustment of geometry parameters of the antenna to ensure both its proper operation in the UWB band and allocation of the notches at the requested frequencies. It is normally carried out by means of parameter sweeps (typically, one parameter at a time) guided by engineering experience. However, handling multiple parameters is not practical this way and can hardly lead to truly optimum results.

Automation of the geometry adjustment process using numerical optimization techniques is highly desirable. Unfortunately, conventional optimization methods (e.g., gradient-based ones) require a large number of objective function evaluations. On the other hand, reliable evaluation of antenna performance can only be achieved by means of fullwave electromagnetic (EM) analysis which is computationally expensive. Therefore, it may be difficult to obtain desired design specifications in reasonable timeframe, especially when conventional optimization techniques are utilized.

The difficulties related to high computational cost of numerical optimization can be addressed by surrogate-based optimization (SBO) techniques [12]-[14]. These methods proved to be very efficient in numerous antenna-related design problems [12], [15]. In SBO, direct optimization of the highfidelity antenna model is replaced by iterative enhancement and re-optimization of its cheaper representation referred to as a surrogate. The surrogate model of antenna structure is usually constructed using coarsely-discretized low-fidelity antenna model and a suitable correction technique [16].

Another possibility is utilization of multiple coarsediscretization models [16]. Variable-fidelity techniques benefit from shifting the optimization burden into the cheaper model with limited accuracy. The fidelity of the model is iteratively increased when approaching to the optimal design, so that normally only a few high-fidelity model evaluations are required to obtain the final design solution. 
In this work, we present a two-stage surrogate-based optimization methodology for fast geometry adjustment of UWB antennas with band-notch capability. In the first stage, a UWB operation of the antenna is obtained by adjusting its relevant parameters using variable-fidelity EM simulation models and response correction techniques. In the second stage, only the parameters responsible for the band-notch are adjusted using sequential approximation optimization, also relying on variable-fidelity EM antenna models. The second stage also exploits correlations between the low- and the highfidelity model responses, so that the discrepancies between them are accounted for at the coarsely-discretized model level by modifying the band-notch specifications. Consequently, the computational cost of design optimization procedure is reduced. The operation and performance of the proposed approach are demonstrated using exemplary UWB monopole antenna with a lower WLAN band-notch realized using complementary rectangular shape split-ring resonator.

\section{DESIGN CASE: UWB MONOPOLE WITH LOWER WLAN BAND-NOTCH}

Consider a UWB monopole antenna with band-notch behavior shown in Fig. 1. The structure is a modification of the design presented in [12] and it consists of a driven element in the form of trapezoid-shape radiator fed through a $50 \mathrm{Ohm}$ microstrip line (both are represented in Fig. 1 using dark shade grey, whereas ground plane is marked using light shade gray). The band-notch behavior is obtained by means of a complementary rectangular-shape split ring resonator implemented in the center of radiator. The antenna is described by two independent vectors of design parameters, i.e., the structure-related ones $\boldsymbol{x}_{a}=\left[\begin{array}{lllll}a_{1} & a_{2} & b_{1} & l & w_{2}\end{array}\right]^{T}$ and the notch-related ones $\boldsymbol{x}_{b}=\left[\begin{array}{llllll}l_{c 1} & l_{c 2} & l_{g} & w_{c 1} & w_{c 2} & g\end{array}\right]^{T}$. The feed line width is set to $w_{1}=1.7$ to ensure $50 \mathrm{Ohm}$ input impedance, whereas the gap between the radiator and the ground plane, and distance of the radiator from the edge of substrate are $d=$ 0.6 and $o=5$, respectively. The unit of all dimensions is $\mathrm{mm}$.

The structure is implemented on $0.762 \mathrm{~mm}$ thick Taconic RF-35 dielectric substrate with permittivity of 3.5 and loss tangent of 0.0018 . The high-fidelity antenna model $\boldsymbol{R}_{f}$ consists of about 2,700,000 hexahedral mesh cells and its average simulation time is 42 minutes. The low-fidelity antenna model $\boldsymbol{R}_{c}$ is constructed using $\sim 240,000$ mesh cells and its evaluation time is 90 seconds. Other simplifications of the $\boldsymbol{R}_{c}$ model include utilization of lossless dielectric and representation of metal as perfect electrical conductor. Both models are implemented in CST Microwave Studio and simulated using its transient solver [17].

The initial antenna design is $\boldsymbol{x}_{a}^{(0)}=\left[\begin{array}{llll}10.0 & 20.0 & 18.0 & 12.0\end{array}\right.$ $40.0]^{T}$ and $\boldsymbol{x}_{b}{ }^{(0)}=\left[\begin{array}{llllll}7.0 & 7.0 & 2.0 & 0.2 & 0.2 & 0.2\end{array}\right]^{T}$. The design objective is to minimize the return loss within the $3.1 \mathrm{GHz}$ to 10.6 GHz frequency band of interest. Simultaneously, it is desired maximize $\left|S_{11}\right|$ above $-5 \mathrm{~dB}$ for the frequencies from $5.1 \mathrm{GHz}$ to $5.3 \mathrm{GHz}$. This range corresponds to the lower WLAN band with about $50 \mathrm{MHz}$ margin on each side.

\section{Design Optimization Methodology}

The design optimization process is divided into two stages: (i) optimization of antenna-only parameters $\boldsymbol{x}_{a}$ to obtain UWB operation, i.e., $\left|S_{11}\right| \leq-10 \mathrm{~dB}$ for $3.1 \mathrm{GHz}$ to $10.6 \mathrm{GHz}$, and (ii) optimization of parameters $\boldsymbol{x}_{b}$ to allocate the notch at required frequencies, here, lower WLAN band. This "decomposition" is possible because of the fact that the band-notch parameters do not significantly affect the UWB antenna operation (except, of course, the frequencies corresponding to the band-notch). Discussed design procedure allows reducing the number of parameters involved in the optimization process to only five or six at a time, depending on the selected component. Consequently, number of EM model evaluations required to converge to the final design is significantly lower than for the multi-parameter design involving all the parameters simultaneously.

\section{A. Antenna Optimization Using Variable-Fidelity EM Models}

At the first stage, the parameter vector $\boldsymbol{x}_{a}$ is optimized to ensure UWB performance of the antenna with the band-notch disabled. The task is to solve

$$
\boldsymbol{x}_{a}^{*}=\arg \min _{\boldsymbol{x}_{a}} U_{a}\left(\boldsymbol{R}_{f}\left(\boldsymbol{x}_{a}\right)\right)
$$

where $U_{a}$ is an objective function encoding performance specifications; $\boldsymbol{x}_{a}{ }^{*}$ is the optimum design to be found. $U_{a}$ is defined as

$$
U_{a}\left(\boldsymbol{R}_{f}\left(\boldsymbol{x}_{a}\right)\right)=\max \left\{\left|S_{11}\right|_{3.1 \mathrm{GHz} \text { to } 10.6 \mathrm{GHz}}\right\}
$$

For the sake of computational efficiency, we utilize surrogate-based optimization (SBO) [13] exploiting the coarsediscretization model $\boldsymbol{R}_{c}$ (cf. Section II). The SBO algorithm is an iterative process

$$
\boldsymbol{x}_{a}^{(i+1)}=\arg \min _{\boldsymbol{x}_{a}} U_{a}\left(\boldsymbol{R}_{s}^{(i)}\left(\boldsymbol{x}_{a}\right)\right)
$$

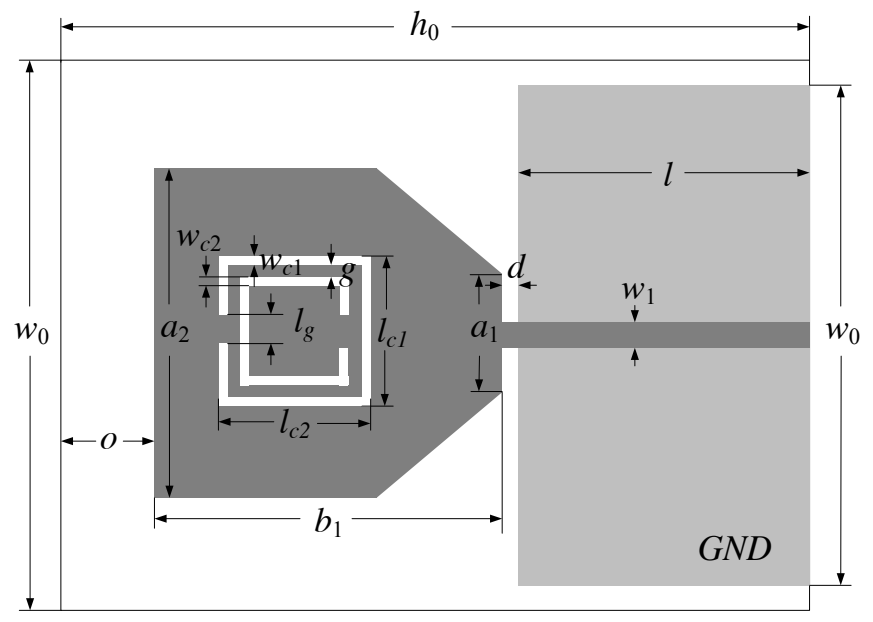

Fig. 1. Geometry of UWB antenna with band-notch at the lower WLAN band. 
where $\boldsymbol{R}_{s}^{(i)}$ is a surrogate model obtained by correcting $\boldsymbol{R}_{c}$, whereas $\boldsymbol{x}^{(i)}, i=0,1, \ldots$, is a sequence of approximated solutions to (1). We utilize an output space-mapping response correction [14] of the form

$$
\boldsymbol{R}_{s}^{(i)}\left(\boldsymbol{x}_{a}\right)=\boldsymbol{R}_{c}\left(\boldsymbol{x}_{a}\right)+\left[\boldsymbol{R}_{f}\left(\boldsymbol{x}_{a}{ }^{(i)}\right)-\boldsymbol{R}_{c}\left(\boldsymbol{x}_{a}{ }^{(i)}\right)\right]
$$

which is suitable for the UWB return loss responses as they are not extremely steep.

\section{B. Allocation of Band-Notch Using Sequential Approximate Optimization}

At this stage, the antenna parameters are fixed (i.e., at $\boldsymbol{x}_{a}{ }^{*}$, as discussed in Section II. $A$ ). The set of variables $\boldsymbol{x}_{b}$ is now optimized to allocate the band-notch in the frequency range from $5.1 \mathrm{GHz}$ to $5.3 \mathrm{GHz}$. The objective function for implementing this requirement will be denoted as $U_{b}$, and the problem at hand is

$$
\boldsymbol{x}_{b}{ }^{*}=\operatorname{argmin}\left\{\boldsymbol{x}_{b}: U_{b}\left(\boldsymbol{R}_{f}\left(\boldsymbol{x}_{b}\right)\right)\right\}
$$

Here, we also utilize the low-fidelity model $\boldsymbol{R}_{c}$ in a sequential approximate optimization process of the following form

$$
\boldsymbol{x}_{b}^{(i+1)}=\arg \min _{\boldsymbol{x}_{b}} U_{b}^{\#}\left(\boldsymbol{G}^{(i)}\left(\boldsymbol{x}_{b}\right)\right)
$$

where $\boldsymbol{G}^{(i)}$ is a linear approximation of $\boldsymbol{R}_{c}$ w.r.t. $\boldsymbol{x}_{b}$ established at $\boldsymbol{x}_{b}{ }^{(i)}$ using $\boldsymbol{R}_{c}\left(\boldsymbol{x}_{b}{ }^{(i)}\right)$ and $n_{b}$ perturbed designs $\left(n_{b}\right.$ being the number of band-notch-related parameters); $\boldsymbol{x}_{b}^{(i)}, i=1,2, \ldots$, are approximations to $\boldsymbol{x}_{b}{ }^{*}$. The objective function $U_{b}{ }^{\#}$ takes into account the misalignment between $\boldsymbol{R}_{c}$ and $\boldsymbol{R}_{f}$ and it is reset after each iteration of (4) based on the discrepancies between the models at the current design, i.e., $\boldsymbol{R}_{f}\left(\boldsymbol{x}_{b}{ }^{(i)}\right)$ and $\boldsymbol{R}_{c}\left(\boldsymbol{x}_{b}{ }^{(i)}\right)$. Exploitation of the correlations between the EM models of various fidelities allow for lowering the cost of the optimization process in a way similar to the adaptively adjusted design specifications technique [18]. A typical difference between the $\boldsymbol{R}_{c}$ and $\boldsymbol{R}_{f}$ responses and modification of design specs is shown in Fig. 2. For fine tuning of $\boldsymbol{x}_{b}$, which is the last iteration of the algorithm, the linear model $\boldsymbol{G}$ is constructed form high-fidelity model perturbations only.

\section{RESULTS}

The UWB antenna of Section II has been optimized using the surrogate-based optimization methodology of Section III. The parameter vector obtained at the first stage of the process is $\boldsymbol{x}_{a}{ }^{*}=\left[\begin{array}{lllll}10.78 & 23.78 & 20.67 & 11.61 & 30.89\end{array}\right]^{T} \mathrm{~mm}$. The computational cost of this stage was only three iterations of (3) involving, in total, 150 evaluations of $\boldsymbol{R}_{c}$ and four evaluations of $\boldsymbol{R}_{f}$. Figure 3 shows the responses of the antenna at the initial design and at $\boldsymbol{x}_{a}{ }^{*}$. It should be noted that the inband return loss of the designed antenna structure are reduced below the level of $-15 \mathrm{~dB}$.

The optimized parameters of the band-notch, $\boldsymbol{x}_{b}{ }^{*}=[11.8$ $\left.\begin{array}{lllll}6.27 & 6.2 & 0.2 & 0.4 & 0.233\end{array}\right]^{T} \mathrm{~mm}$ have been obtained in five iterations of (6) and an additional fine-tuning iteration of (6) using high-fidelity evaluations model only. Figure 4 shows the initial and the final return loss responses of the antenna with the optimized band-notch. The obtained results indicate that final design fulfills the design specification. It can be also observed that introduction of complementary split-ring resonator to the radiator has minor impact on the antenna return loss characteristics so that the components of the vector $\boldsymbol{x}_{b}$ can be adjusted with the antenna parameters fixed at $\boldsymbol{x}_{a}{ }^{*}$.

The total numerical cost of the second design stage was 35 evaluations of $\boldsymbol{R}_{c}$ model and 12 evaluations of $\boldsymbol{R}_{f}$ one. Thus, the total aggregated cost of the surrogate-based design optimization procedure corresponds to only 23 evaluations of the high-fidelity model. This cost is considered low, especially taking into account the relatively large number of eleven antenna design variables.

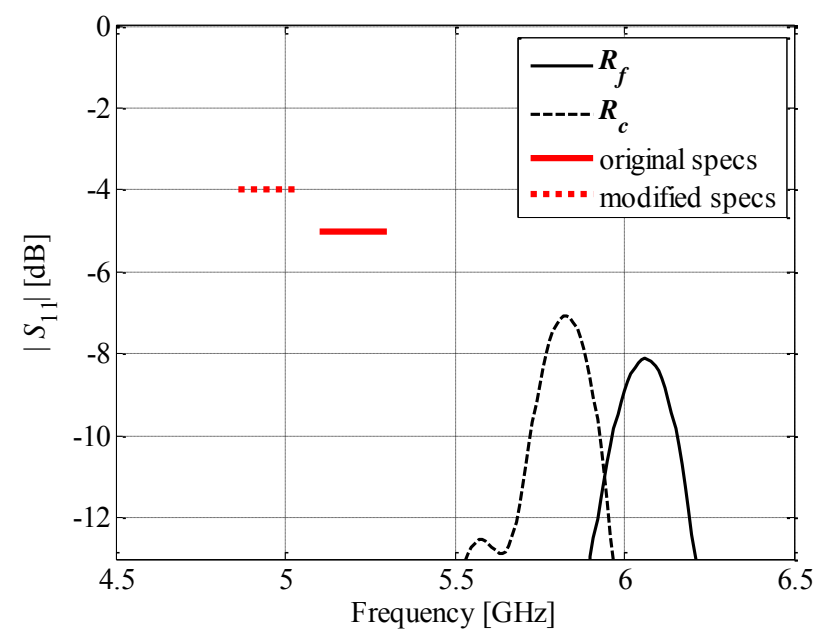

Fig. 2. Responses of the band-notch for high- (-) and low-fidelity (- - -) model at the initial design of $\boldsymbol{x}_{b}$. The modified design specifications for the band-notch account for the discrepancies between the low- and high-fidelity models. The specs modifications are reset after each iteration of (4).

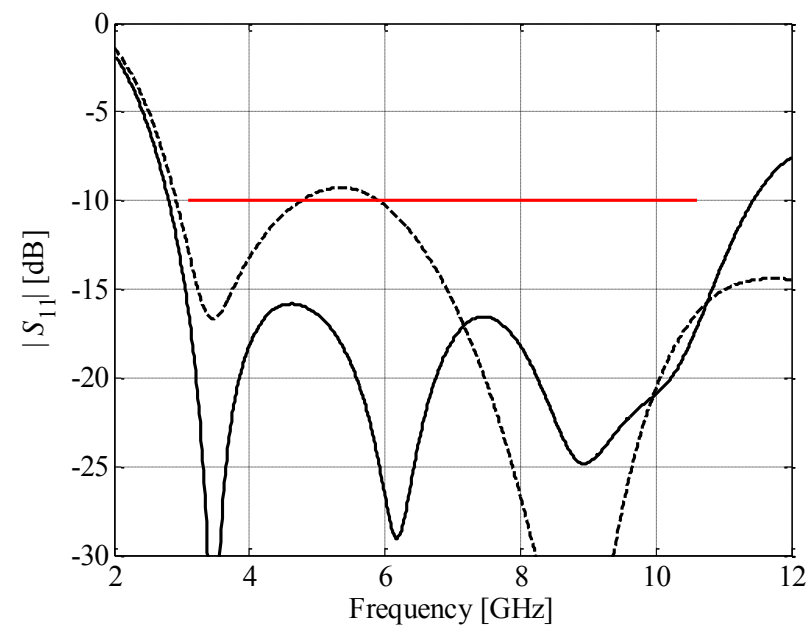

Fig. 3. Return loss of the UWB monopole antenna of Fig. 1 at the initial design $\boldsymbol{x}_{a}{ }^{(0)}(---)$ and the optimized design $(-) \boldsymbol{x}_{a}{ }^{*}$. UWB design specifications are marked with thick horizontal line. 


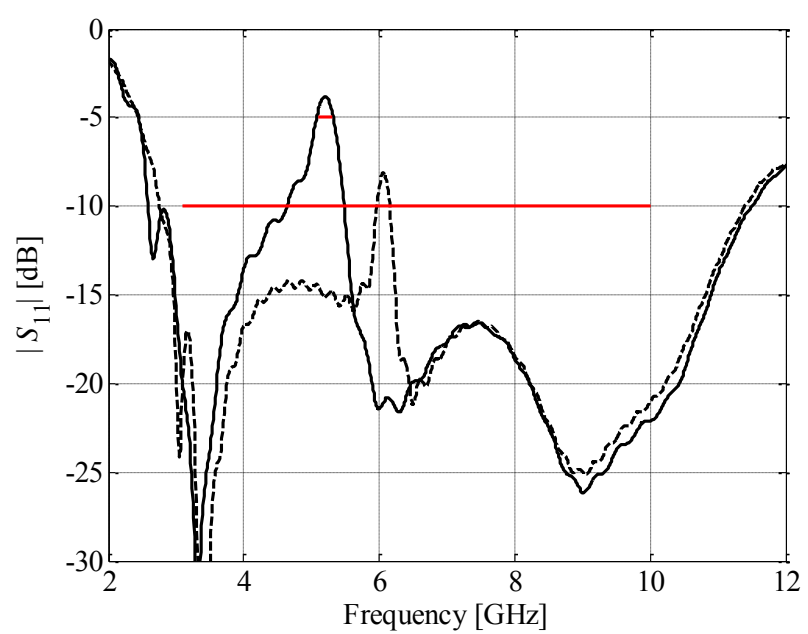

Fig. 4. Return loss response of the UWB monopole of Fig. 1 with implemented band-notch capability: (a) at the initial design (- - -), and (b) with relevant geometry parameters optimized (-). UWB and band-notch design specifications marked with long and short thick horizontal lines, respectively.

\section{CONCLUSION}

A computationally efficient methodology for design optimization of band-notch UWB antennas has been presented. Our approach exploits surrogate-based optimization (SBO) involving variable-fidelity EM antenna models, as well as problem decomposition into two independent design stages, i.e., design of the antenna itself and the adjustment bandnotch-related parameters. At each stage, a different SBO approach is utilized, which is tailored to the specific challenges of the problem at hand (output space mapping for antenna design, and design specifications adaptation for bandnotch parameter optimization). The final design satisfying the prescribed specifications is obtained at the total cost corresponding to only 23 evaluations of the high-fidelity antenna model.

\section{ACKNOWLEDGMENT}

The authors would like to thank Computer Simulation Technology AG, Darmstadt, Germany, for making CST Microwave Studio available. This work was supported in part by the Icelandic Centre for Research (RANNIS) Grant 130450051, by the Interuniversity Attraction Poles Programme BESTCOM initiated by the Belgian Science Policy Office, and by the Fund for Scientific Research in Flanders (FWOVlaanderen).

\section{REFERENCES}

[1] M. Bod, H.R. Hassani, and M.M.S Taheri, "Compact UWB Printed Slot Antenna With Extra Bluetooth, GSM, and GPS Bands," IEEE Ant. Wireless Prop. Lett., vol. 11, pp. 531-534, 2012.
[2] N. Chahat, M. Zhadobov, R. Sauleau, K. Ito, "A Compact UWB Antenna for On-Body Applications," IEEE Trans. Ant. Prop., vol. 59, no. 4, pp. 1123-1131, 2011.

[3] H.R. Khaleel, H.M. Al-Rizzo, D.G. Rucker, S. Mohan, "A Compact Polyimide-Based UWB Antenna for Flexible Electronics," IEEE Ant. Wireless Prop. Lett., vol. 11, pp. 564$567,2012$.

[4] A. Dumoulin, M. John, M.J. Ammann, P. McEvoy, "Optimized Monopole and Dipole Antennas for UWB Asset Tag Location Systems," IEEE Trans. Ant. Prop., vol. 60, no. 6, pp. 2896-2904, 2012.

[5] D. Gibbins, M. Klemm, I. Craddock, A. Preece, J Leendertz, R. Benjamin, "Design of a UWB wide-slot antenna and a hemispherical array for breast imaging," European Conf. Ant. Prop., pp. 2967-2970, 2009.

[6] P. Gao, S. He, X. Wei, Z. Xu, N. Wang, Y. Zheng, "Compact Printed UWB Diversity Slot Antenna With 5.5-GHz BandNotched Characteristics," IEEE Ant. Wireless Prop. Lett., vol.13, pp. 376-379, 2014.

[7] K.S. Ryu, and A.A. Kishk, "UWB antenna with single or dual band-notches for lower WLAN band and upper WLAN band," IEEE Trans. Ant. Prop., vol. 57, no. 12, pp. 3942-3950, 2009.

[8] A. C. Shagar, and R. S. D. Wahidabanu, "Compact slot antenna with band-notch characterization for multifunctional communication systems," Microwave Optical Tech. Lett., vol. 55, pp. 218-223, Jan. 2013.

[9] T. Li, H. Zhai, G. Li, L. Li, and C. Lian,"Compact UWB bandnotched antenna design using interdigital capacitance loading loop resonator," IEEE Antennas Wireless Prop. Lett., vol. 11, pp. 724-727, 2012.

[10] D. Sarkar, K.V. Srivastava, and K. Saurav, "A compact microstrip-fed triple band-notched UWB monopole antenna," IEEE Antennas Wireless Prop. Lett., vol. 13, pp. 396-399, 2014.

[11] D.T. Nguyen, D.H. Lee, and H.C. Park, "Very compact printed triple band-notched UWB antenna with quarter-wavelength slots," IEEE Antennas Wireless Prop. Lett., vol. 11, pp. 411-414, 2012.

[12] S. Koziel, A. Bekasiewicz, I. Couckuyt, and T. Dhaene, "Efficient multi-objective simulation-driven antenna design using co-kriging," IEEE Trans. Antennas Prop., vol. 62, no. 11, pp. 5900-5905, 2014.

[13] M.B. Yelten, T. Zhu, S. Koziel, P.D. Franzon, and M.B. Steer, "Demystifying surrogate modeling for circuits and systems," IEEE Circuits and Systems Magazine, vol. 12, no. 1, pp. 45-63, 2012.

[14] S. Koziel, Q.S. Cheng, J.W. Bandler, "Space mapping," IEEE Microwave Magazine, vol. 9, no. 6, pp. 105-122, 2008.

[15] A. Bekasiewicz, and S. Koziel, "Structure and ComputationallyEfficient Simulation-Driven Design of Compact UWB Monopole Antenna,” IEEE Ant. Wireless Prop. Lett., 2015.

[16] S. Koziel and X.S. Yang (Eds.), "Computational optimization, methods and algorithms," Series: Studies in Computational Intelligence, vol. 356, Springer, 2011.

[17] CST Microwave Studio, ver. 2013, CST AG, Bad Nauheimer Str. 19, D-64289 Darmstadt, Germany, 2013.

[18] S. Koziel and S. Ogurtsov, "Rapid optimization of omnidirectional antennas using adaptively adjusted design specifications and kriging surrogates," IET Microwaves, Ant. Prop., vol. 7, no. 15, pp. 1194-1200, 2013. 